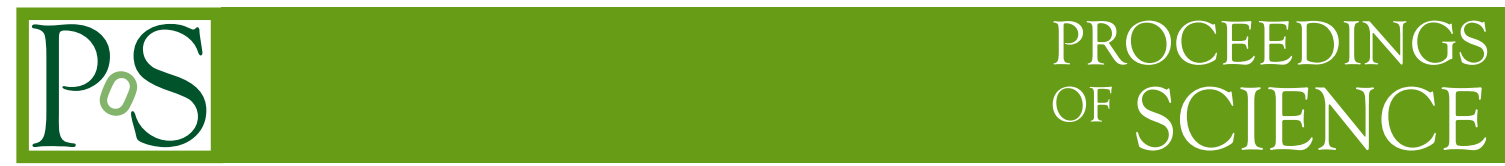

\title{
The hunt for axions
}

\section{Andreas Ringwald}

Deutsches Elektronen-Synchrotron DESY

Notkestr. 85

D-22607 Hamburg

E-mail: andreas.ringwaldedesy.de

Many theoretically well-motivated extensions of the Standard Model of particle physics predict the existence of the axion and further ultralight axion-like particles. They may constitute the mysterious dark matter in the universe and solve some puzzles in stellar and high-energy astrophysics. There are new, relatively small experiments around the globe, which started to hunt for these elusive particles and complement the search for physics beyond the Standard Model at the Large Hadron Collider.

XVI International Workshop on Neutrino Telescopes,

2-6 March 2015

Palazzo Franchetti - Istituto Veneto, Venice, Italy 


\section{Introduction}

In particle physics, we are in the comfortable situation that we have a theory - the Standard Model (SM) of particle physics - which describes all known particles and their interactions to a remarkable precision. On the other hand, astronomical observations tell us that only about fifteen percent of the matter in the universe is constituted by the known particles. It is one of the most urgent problems of fundamental physics to understand the nature of the remaining eighty-five percent of matter.

Theoretical particle physicists have been very imaginative to propose dark matter candidates spanning a huge range in mass and in strength of their interactions. Among those candidates two particular ones stick out both because of their appealing physics case and the variety of experimental probes: the neutralino - the lightest supersymmetric partner of the SM neutral gauge bosons and the neutral Higgses - as a typical weakly interacting massive particle (WIMP) and the axion - the pseudo Nambu-Goldstone boson arising from the breaking of a global symmetry postulated in order to solve the strong CP problem - as a typical very weakly interacting slim (in the sense of ultralight) particle (WISP). We will devote our attention in this short review to the latter species (for more extended reviews, see Refs. [1,2]).

\section{Nambu-Goldstone bosons as natural WISP candidates}

WISPs occur naturally in SM extensions featuring new global $U(1)$ symmetries which are spontaneously broken by a hidden Higgs mechanism at a symmetry breaking scale $v_{h}$ much larger than the electroweak symmetry breaking scale, $v=246 \mathrm{GeV}$. In this case, the field $a(x)$ in the phase of the expansion of the hidden complex Higgs field about its vacuum expectation value (vev), $H_{h}(x)=\frac{1}{\sqrt{2}}\left(v_{h}+h_{h}(x)\right) \mathrm{e}^{i a(x) / v_{h}}$, has a flat potential, $V(a) \equiv$ const., and is thus massless, while the field excitation $h_{h}(x)$ of the modulus has a large mass $m_{h} \propto v_{h} \gg v$. Moreover, at energies below the electroweak scale, the interactions of the so-called Nambu-Goldstone boson $a$ with the SM particles - gauge bosons (gluons with field strength $G$ and photons with field strength $F$ ) and fermions $f$ (light quarks and leptons) - are suppressed by the inverse of the large symmetry breaking scale,

$$
\mathscr{L}=\frac{1}{2} \partial_{\mu} a \partial^{\mu} a-\frac{\alpha_{s}}{8 \pi} C_{a g} \frac{a}{f_{a}} G_{\mu v}^{c} \tilde{G}^{c, \mu v}-\frac{\alpha}{8 \pi} C_{a \gamma} \frac{a}{f_{a}} F_{\mu v} \tilde{F}^{\mu v}+\frac{1}{2} \frac{C_{a f}}{f_{a}} \partial_{\mu} a \bar{\psi}_{f} \gamma^{\mu} \gamma_{5} \psi_{f},
$$

with model-dependent dimensionless coupling coefficients $C_{a j}$ and a decay constant $f_{a} \propto v_{h}$.

In models with $C_{a g} \neq 0$, the so-called strong CP problem is solved by non-perturbative QCD dynamics: the effective potential for $a$ has then an absolute minimum at $a=0$ and therefore the effective theta parameter - proportional to the vev of $a$-vanishes [3]. The corresponding NambuGoldstone boson is called the axion [4, 5]. Strictly speaking, it is a pseudo Nambu-Goldstone boson: it gets a small mass, $V(a)=\frac{1}{2} m_{a}^{2} a^{2}+\mathscr{O}\left(a^{4}\right)$, due to the same non-perturbative QCD effects,

$$
m_{a}=\frac{m_{\pi} f_{\pi}}{f_{a} / C_{a g}} \frac{\sqrt{m_{u} m_{d}}}{m_{u}+m_{d}} \simeq 6 \mu \mathrm{eV} \times\left(\frac{10^{12} \mathrm{GeV}}{f_{a} / C_{a g}}\right) .
$$

Here $m_{\pi}$ and $f_{\pi}$ are the neutral pion mass and decay constant, and $m_{u}$ and $m_{d}$ are the masses of the light quarks. There may be further axion-like particles (ALPs) which arise as Nambu-Goldstone 
bosons from the breaking of further well motivated global symmetries and which have no coupling to gluons, but a non-zero coupling to photons and/or to light quarks and leptons [6]. In fact, string theory suggests a plenitude of such ALPs $[7,8,9,10]$.

\section{Axion/ALP dark matter?}

For large symmetry breaking scales, axions and ALPs have lifetimes much longer than the age of the universe and interact extremely weakly with the SM particles, qualifying them as dark matter candidates. Indeed, they are produced in the early universe via the vacuum realignment mechanism as a coherent state of many, extremely non-relativistic (and thus extremely cold) dark matter particles in the form of a classical, spatially coherent oscillating field $[11,12,13]$. Today's (time $t_{0}$ ) fraction of axion or ALP dark matter produced via the vacuum realignment mechanism is proportional to the average field amplitude squared, $\left\langle a^{2}\right\rangle \equiv f_{a}^{2}\left\langle\theta_{a}^{2}\right\rangle$, at the time when the oscillations started, $t_{\mathrm{osc}} \simeq(3 / 2) m_{a}^{-1}\left(t_{\mathrm{osc}}\right),[14]$

$$
R_{a} \equiv \frac{\rho_{a}}{\rho_{\mathrm{DM}}}\left(t_{0}\right) \simeq 0.2 \sqrt{\frac{m_{a}\left(t_{0}\right)}{\mathrm{eV}}} \sqrt{\frac{m_{a}\left(t_{0}\right)}{m_{a}\left(t_{\mathrm{osc}}\right)}}\left(\frac{f_{a}}{10^{11} \mathrm{GeV}}\right)^{2}\left\langle\theta_{a}^{2}\right\rangle .
$$

Here, the indicated time-dependence of the mass arises from its temperature dependence, $m_{a}(t) \equiv$ $m_{a}(T(t))$, taking into account possible plasma effects. From (3.1) it follows, that an appreciable fraction of light axion/ALP dark matter is only expected for sufficiently large symmetry breaking scale, $f_{a} \gtrsim 10^{10 \div 12} \mathrm{GeV}$ and correspondingly small couplings to SM particles (see Fig. 1).

\section{Hints on axion/ALPs from astrophysics}

\subsection{Axion/ALP energy losses of stars?}

Stringent bounds on the axion or ALP couplings to photons and electrons in a wide mass range have been established from observations of stars in globular-clusters (GCs) [16]. These are relying on the fact that number counts of stars in particular branches of the color-magnitude diagram of GCs allow for detailed tests of stellar evolution. Recently, several authors have confronted new data with improved theoretical predictions and found hints for anomalous energy losses.

A recent state-of-the-art analysis of horizontal branch, i.e. helium burning, stars in a large sample of 39 Galactic GCs, exploiting modern stellar models, and taking into account the substantial dependence of the predictions on the He mass fraction $Y$, found a slight indication of additional losses which may be accounted by Primakoff-like axion/ALP emission in the Coulomb field of a charged particle, $\gamma+Z e \rightarrow Z e+a$, if the photon coupling is in the range [17]

$$
g_{a \gamma} \equiv \frac{\alpha}{2 \pi} \frac{C_{a \gamma}}{f_{a}}=4.5_{-1.6}^{+1.2} \times 10^{-11} \mathrm{GeV}^{-1}, \quad \text { for } m_{a} \lesssim \mathrm{keV} .
$$

Conservatively, the authors of this analysis determine also an upper bound, $g_{a \gamma}<6.6 \times 10^{-11} \mathrm{GeV}^{-1}$, at $95 \%$ confidence level (CL), which represents the strongest limit on $g_{a \gamma}$ for a wide mass range.

Clearly, the result (4.1) gives only a marginal hint for the existence of the QCD axion or an ALP. In fact, at two sigma it is still compatible with the SM, $g_{a \gamma}=0$. However, there are other 


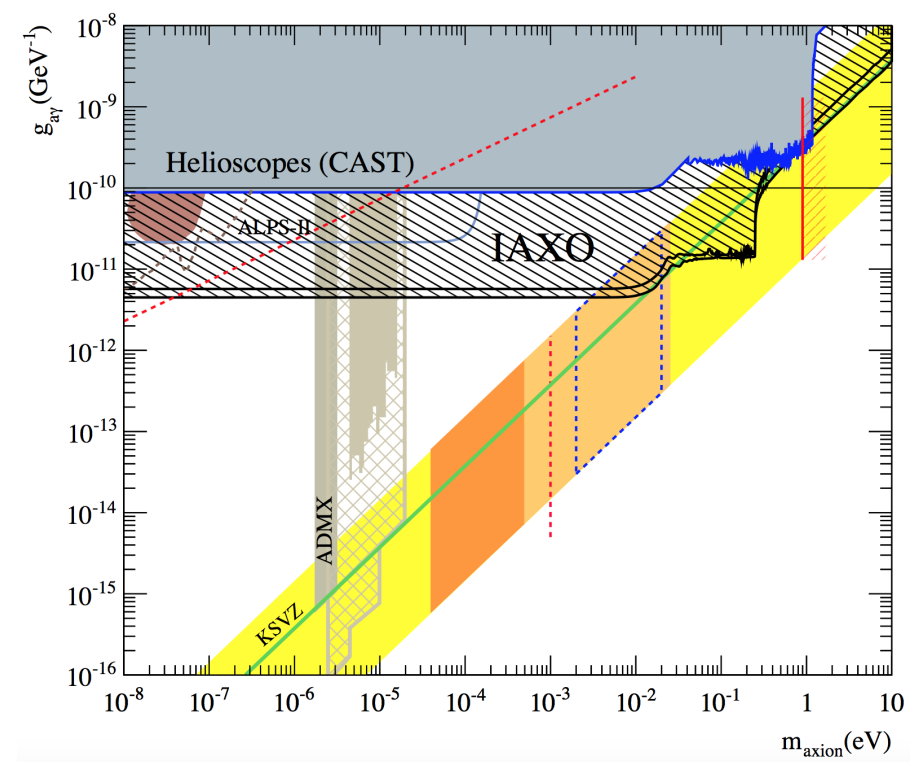

Figure 1: Current experimental constraints on the photon coupling of an axion or ALP [15]. Theoretically and astrophysically favored regions are shown for axions within the yellow model band (classical axion window in dark orange, mixed axion-WIMP DM in light orange, RG and WD cooling hint within the area surrounded by the dashed blue line) and for ALPs (brown dashed line for transparency hint, below red dashed diagonal line for ALP cold DM). Future prospects of ALPS II (above light blue line), IAXO (dashed black region), and ADMX (dashed brown region) are also shown.

mismatches between theory and observations which may also be seen as slight indications for the existence of a Nambu-Goldstone boson with such a coupling to the photon. One of them is the fact that the ratio of blue to red supergiants (SGs) is smaller than predicted by stellar evolution models. Moreover, the blue SGs appear to be less blue than expected [18]. This effect could also be explained by an axion/ALP with $g_{a \gamma}=$ few $\times 10^{-11} \mathrm{GeV}^{-1}$ [19].

Interestingly enough, also Red Giants (RGs) in GCs mildly prefer additional energy losses, in this case, however, due to axion/ALP emission via bremsstrahlung of axions or ALPs, $e+Z e \rightarrow$ $Z e+e+a$, pointing to an electron coupling in the range [20,21]

$$
g_{a e} \equiv \frac{C_{a e} m_{e}}{f_{a}}=1.8_{-0.8}^{+0.6} \times 10^{-13}, \quad \text { for } m_{a} \lesssim \mathrm{keV} .
$$

Still, at $\sim 2 \sigma$, the result is compatible with the SM, $g_{a e}<4.3 \times 10^{-13}(95 \% \mathrm{CL})$.

Another astrophysical observable probing the electron coupling is the luminosity function of white dwarfs (WDs). Intriguingly, recent analyses, based on detailed WD cooling treatment and new data, find weak evidence that the WD luminosity function fits better with a new energy-loss channel that can be interpreted in terms of axion/ALP losses via bremsstrahlung in electron-ion or electron-electron collsions, requiring an electron coupling in the range $g_{a e}=1.0_{-0.2}^{+0.2} \times 10^{-13}$, for $m_{a} \lesssim \mathrm{keV}[22,23]$. This is consistent with the axion/ALP explanation of the apparent excessive energy loss of RGs, cf. Eq. (4.2). However, also in the WD case the evidence is still rather weak: at $2 \sigma$ the WD luminosity function is consistent with the SM, $g_{a e}<2.3 \times 10^{-13}(95 \% \mathrm{CL}$ ). 
Last, but not least, there is also a hint on extra energy losses of the neutron star in Cas A: its surface temperature measured over 10 years reveals an unusually fast cooling rate. This may be interpreted as a hint on extra cooling by axion/ALP due to nucleon bremsstrahlung, $N+N \rightarrow$ $N+N+a$, requiring a coupling to the neutron of size [24]

$$
g_{a n} \equiv \frac{C_{a n} m_{n}}{f_{a}} \sim 4 \times 10^{-10}, \quad \text { for } m_{a} \lesssim \mathrm{MeV} .
$$

Still, this can also be seen as an approximate upper limit on this coupling. In fact, recently it was pointed out that the more rapid cooling of the superfluid core in the neutron star may also arise from a phase transition of the neutron condensate into a multicomponent state [25].

Clearly, the evidence for each of the anomalous energy loss hints is very weak. But nevertheless, a unified picture seems to emerge: just one light, $m_{a} \lesssim \mathrm{keV}$, pseudo Nambu-Goldstone boson with $f_{a} \sim 10^{8} \mathrm{GeV}, C_{a \gamma} \sim 1$, and $C_{a e} \sim C_{a n} \sim 10^{-2}$, is required to explain at the same time the indications from the three quite different stellar energy loss channels. Intriguingly, in LARGE volume, $\mathscr{V} \gg 1$, string compactifications the decay constant of closed string ALPs is generically much smaller than the Planck scale, $f_{a} \sim M_{\mathrm{Pl}} / \sqrt{\mathscr{V}}$, and the matter coupling coefficients $C_{a f}$ of closed string ALPs are generically suppressed by a factor $\alpha \sim 10^{-2}$ in comparison to the photon coupling coefficient $C_{a \gamma}[10]$, realising the required properties.

\subsection{Photon-ALP oscillations in astrophysical magnetic fields?}

Photon-ALP oscillations in large-scale interstellar as well as in local magnetic fields associated with astrophysical objects provide another sensitive probe for ALPs complementary to Primakoff conversion in stars. However, this probe is confined to very low masses,

$$
\left|m_{a}^{2}-\omega_{\mathrm{pl}}^{2}\right| \ll 2 g_{a \gamma} B E \simeq 2.5 \times \mathrm{neV}^{2}\left(\frac{g_{a \gamma}}{10^{-11} \mathrm{GeV}^{-1}}\right)\left(\frac{B}{\mu \mathrm{G}}\right)\left(\frac{E}{\mathrm{GeV}}\right),
$$

where $\omega_{\mathrm{pl}} \simeq 4 \times 10^{11} \mathrm{eV} \sqrt{n_{e} / \mathrm{cm}^{3}}$ is the plasma frequency in terms of the electron density in the medium, while $B$ is the magnetic field strength and $E$ is the photon/axion/ALP energy.

In a galactic core-collapse supernova (SN), ALPs would be emitted via the Primakoff process, and oscillate into gamma rays in the magnetic field of the Milky Way. The lack of a gammaray signal in the GRS instrument of the SMM satellite in coincidence with the observation of the neutrinos emitted from SN1987A therefore provides a strong bound on their coupling to photons, cf. [26, 27]. Recently, this bound has been revisited and the underlying physics has been brought to the current state-of-the-art, as far as modelling of the supernova and the Milky-Way magnetic field are concerned, resulting in the limit $g_{a \gamma}<5.3 \times 10^{-12} \mathrm{GeV}^{-1}$, for $m_{a} \lesssim 4.4 \times 10^{-10} \mathrm{eV}$ [28]. Therefore, the mass window for ALPs explaining the HB energy loss shrinks to $\mathrm{neV} \lesssim \mathrm{m}_{\mathrm{a}} \lesssim \mathrm{keV}$.

Gamma-ray spectra from distant active galactic nuclei (AGN) should show an energy and redshift-dependent exponential attenuation, $\exp (-\tau(E, z))$, due to $e^{+} e^{-}$pair production off the extragalactic background light (EBL) - the stellar and dust-reprocessed light accumulated during the cosmological evolution following the era of re-ionization. However, a number of authors [29, $30,31]$ have noted that the observed spectra seem to point to an anomalous transparency of the universe for gamma-rays at large optical depth, $\tau \gtrsim 2$, although with evidence below two sigma, if one takes into account systematic effects (EBL spectrum, intrinsic source spectra, etc.) [32]. This 
may be explained by photon $\leftrightarrow$ ALP oscillations: the conversion of gamma rays into ALPs in the magnetic fields around AGNs or in the intergalactic medium, followed by their unimpeded travel towards our galaxy and the consequent reconversion into photons in the (inter)galactic magnetic fields, requiring an ALP with $g_{a \gamma} \gtrsim 10^{-(11 \div 12)} \mathrm{GeV}^{-1}$, for $m_{a} \lesssim 10^{-7} \mathrm{eV}[29,33,34,35,36]$.

Intriguingly, a similar value of the photon coupling is required also for a possible explanation of the rapidly varying very high energy $(E>50 \mathrm{GeV})$ emission from the flat spectrum radio quasar PKS 1222+216 which represents a challenge for standard blazar scenarios: in the latter one is forced to invoke the existence of a very compact emitting region at a large distance from the jet base, in order to avoid absorption of gamma rays in the dense ultraviolet radiation field of the broad line region. In ref. [37] it was shown that one can also use a standard blazar model for PKS $1222+216$ where gamma rays are produced close to the central engine, if one assumes that inside the source photons can oscillate into ALPs. Moreover, the required photon coupling overlaps with the preferred region from the anomalous energy losses of helium burning starts in globular clusters.

Finally, it was found that observed soft X-ray excesses in many galaxy clusters may be explained by the conversion of a cosmic ALP background (CAB) radiation, corresponding to an effective number $\triangle N_{\text {eff }}$ of extra neutrinos, into photons in the cluster magnetic fields [38, 39, 40]. This explanation requires that the $\mathrm{CAB}$ spectrum is peaked in the soft X-ray region and that the ALP coupling and mass satisfy $g_{a \gamma} \gtrsim(1 \div 2) \times 10^{-13} \mathrm{GeV}^{-1} \sqrt{0.5 / \triangle N_{\mathrm{eff}}}$, for $m_{a} \lesssim 10^{-12} \mathrm{eV}$.

\section{The experimental hunt for the axion and ALPs}

We have seen that there is a strong physics case for the axion and other ALPs arising as NambuGoldstone bosons from the breaking of a symmetry at a scale $f_{a} \sim 10^{8 \div 12} \mathrm{GeV}$. Fortunately, such WISPs are in reach of a number of experiments which are presently carried out or being set up.

\subsection{Light-shining-through-a-wall searches}

Light-Shining-Through-Wall (LSW) experiments aim both for production and detection of axions and ALPs in the laboratory. This is done by sending laser photons along a strong magnetic field, allowing for their conversion into axions or ALPs, towards a blocking wall, behind of which the latter may then reconvert, again in a strong magnetic field, into photons, the latter being susceptible to detection (for a review, see [41]). The Any Light Particle Search (ALPS) experiment at DESY and OSQAR at CERN share currently the best sensitivity of LSW experiments [42, 43]. ALPS II [44] proposes to use 10+10 straightened HERA magnets, a high-power laser system, a superconducting low-background detector and the pioneering realization of an optical regeneration cavity $[45,46]$. It aims to crucially test the ALP explanation of the excessive helium burning star energy loss and of the anomalous cosmic gamma ray transparency, cf. Fig. 1.

\subsection{Helioscope searches}

Helioscopes aim to detect solar axions and ALPs produced by their conversion into photons inside of a strong magnet pointing towards the Sun [47]. The CERN Axion Solar Telescope (CAST), employing an LHC dipole test magnet, sets currently the best helioscope limit [48, 49]. A proposed next-generation axion helioscope, dubbed the International Axion Observatory (IAXO), envisions a dedicated superconducting toroidal magnet with much bigger aperture than CAST, a detection 
system consisting of large X-ray telescopes coupled to ultra-low background X-ray detectors, and a large, robust tracking system [50]. It aims at the sensitivity shown in Fig. 1. It will crucially test the axion explanation of the energy losses of helium burnng stars, red giants, white dwarfs and the neutron star in Cas A, and of the ALP explanation of the anomalous cosmic gamma ray transparency. Futhermore, it scratches also the parameter region required to explain the soft X-ray excess from galaxy clusters.

\subsection{Direct axion/ALP dark matter searches}

\subsubsection{Haloscopes}

Haloscopes directly search for galactic halo dark matter axions and ALPs in the laboratory via their coupling to the photon. Currently, the most sensitive ones exploit electromagnetic cavites placed in a strong magnet [47]. They aim for the detection of electromagnetic power arising from the conversion of dark matter axions or ALPs into real photons, with frequency $v=m_{a} /(2 \pi)=$ $0.24 \mathrm{GHz} \times\left(m_{a} / \mu \mathrm{eV}\right)$. The best sensitivity is reached on resonance, the power output then being proportional to the quality factor of the cavity. The Axion Dark Matter eXperiment (ADMX) has indeed reached recently the sensitivity to probe axion dark matter [51] (see Fig. 1). Further microwave cavity based haloscope opportunities in complementary mass ranges may arise from recycling available microwave cavities and magnets at accelerator laboratories [52].

Other new concepts for haloscopes are also being investigated. A microwave Fabry-Perot resonator in a spatially varying magnetic field may be exploited to search for axion/ALP dark matter with masses above $40 \mu \mathrm{eV}$ [53]. Converted photons from axion/ALP dark matter could be focused in a manner similar to a dish antenna, allowing for broad-band searches [54].

\subsubsection{Magnetic resonance searches}

Axion dark matter produced via the misalignment mechanism gives all nucleons oscillating electric dipole moments (EDMs). These EDMs cause the precession of nuclear spins in a nucleon spin polarized sample in the presence of an electric field. The resulting transverse magnetisation can be searched for exploiting magnetic resonance (MR) techniques [55]. The aim of the corresponding Cosmic Axion Spin Precession Experiment (CASPEr) in Mainz is to probe axion dark matter in the anthropic window, corresponding to GUT to Planck scale symmetry breaking scale $f_{a} \sim 10^{15 \div 18} \mathrm{GeV}$, complementary to the classic axion window probed by ADMX.

The axion/ALP nucleon coupling will also lead to a spin precession about the axion/ALP DM wind, even without the presence of an electric field. Therefore, CASPEr can also be exploited to search for the magnetisation due to this effect [56]. Unfortunately, in this case the projected sensitivity does not reach the axion prediction.

Finally, also the axion/ALP electron coupling $[57,58]$ will lead to a spin precession about the axion/ALP DM wind. The QUAX (QUaerere AXions) experiment in preparation in Italy by INFN aims to exploit magnetic resonance (MR) inside a magnetized material [59]. Because of the higher Larmor frequency of the electron, it extends the sensitivity to higher masses. 


\section{Summary}

There is a strong physics case for the axion and ALPs. They occur naturally in many theoretically appealing ultraviolet completions of the SM. They are dark matter candidates and can explain the hints for an excessive energy loss of stars and for an anomalous transparency of the universe for $\mathrm{TeV}$ photons. A significant portion of their parameter space will be tackeled in this decade by experiments. Stay tuned!

\section{References}

[1] J. Jaeckel and A. Ringwald, "The Low-Energy Frontier of Particle Physics," Ann. Rev. Nucl. Part. Sci. 60 (2010) 405 [arXiv:1002.0329 [hep-ph]].

[2] A. Ringwald, "Exploring the Role of Axions and Other WISPs in the Dark Universe," Phys. Dark Univ. 1 (2012) 116 [arXiv:1210.5081 [hep-ph]].

[3] R. D. Peccei and H. R. Quinn, "CP Conservation in the Presence of Instantons," Phys. Rev. Lett. 38 (1977) 1440.

[4] S. Weinberg, “A New Light Boson?,” Phys. Rev. Lett. 40 (1978) 223.

[5] F. Wilczek, "Problem of Strong P and T Invariance in the Presence of Instantons," Phys. Rev. Lett. 40 (1978) 279.

[6] A. G. Dias et al., "The Quest for an Intermediate-Scale Accidental Axion and Further ALPs," JHEP 1406 (2014) 037 [arXiv:1403.5760 [hep-ph]].

[7] E. Witten, "Some Properties of O(32) Superstrings," Phys. Lett. B 149 (1984) 351.

[8] J. P. Conlon, “The QCD axion and moduli stabilisation,” JHEP 0605 (2006) 078 [hep-th/0602233].

[9] A. Arvanitaki et al., "String Axiverse," Phys. Rev. D 81 (2010) 123530 [arXiv:0905.4720 [hep-th]].

[10] M. Cicoli, M. Goodsell and A. Ringwald, "The type IIB string axiverse and its low-energy phenomenology," JHEP 1210 (2012) 146 [arXiv:1206.0819 [hep-th]].

[11] J. Preskill et al., "Cosmology of the Invisible Axion,” Phys. Lett. B 120 (1983) 127.

[12] L. F. Abbott and P. Sikivie, "A Cosmological Bound on the Invisible Axion," Phys. Lett. B 120 (1983) 133.

[13] M. Dine and W. Fischler, “The Not So Harmless Axion,” Phys. Lett. B 120 (1983) 137.

[14] P. Arias et al., "WISPy Cold Dark Matter," JCAP 1206 (2012) 013 [arXiv:1201.5902 [hep-ph]].

[15] J. K. Vogel et al. [IAXO Collaboration], "The Next Generation of Axion Helioscopes: The International Axion Observatory (IAXO)," Phys. Procedia 61 (2015) 193.

[16] G. G. Raffelt, "Stars as laboratories for fundamental physics: The astrophysics of neutrinos, axions, and other weakly interacting particles," Chicago, USA: Univ. Pr. (1996) 664 p.

[17] A. Ayala et al., "Revisiting the bound on axion-photon coupling from Globular Clusters," Phys. Rev. Lett. 113 (2014) 191302 [arXiv:1406.6053 [astro-ph.SR]].

[18] K. B. W. McQuinn et al., "Observational Constraints on Red and Blue Helium Burning Sequences," Astrophys. J. 740 (2011) 48 [arXiv:1108.1405 [astro-ph.CO]]. 
[19] G. Carosi et al., "Probing the axion-photon coupling: phenomenological and experimental perspectives. A snowmass white paper," arXiv:1309.7035 [hep-ph].

[20] N. Viaux et al. "Neutrino and axion bounds from the globular cluster M5 (NGC 5904)," Phys. Rev. Lett. 111 (2013) 231301 [arXiv:1311.1669 [astro-ph.SR]].

[21] G. Raffelt, “Astrophysical and Cosmological Axion Limits,” talk at IBS-MultiDark Joint Focus Program, Daejeon, Korea, 10-21 October 2014.

[22] J. Isern, E. Garcia-Berro, S. Torres and S. Catalan, "Axions and the cooling of white dwarf stars," Astrophys. J. 682 (2008) L109 [arXiv:0806.2807 [astro-ph]].

[23] M. M. Miller Bertolami, B. E. Melendez, L. G. Althaus and J. Isern, "Revisiting the axion bounds from the Galactic white dwarf luminosity function,” JCAP 1410 (2014) 069.

[24] L. B. Leinson, “Axion mass limit from observations of the neutron star in Cassiopeia A,” JCAP 1408 (2014) 031 [arXiv:1405.6873 [hep-ph]].

[25] L. B. Leinson, "Superfluid phases of triplet pairing and rapid cooling of the neutron star in Cassiopeia A,” Phys. Lett. B 741 (2015) 87 [arXiv:1411.6833 [astro-ph.SR]].

[26] J. W. Brockway, E. D. Carlson and G. G. Raffelt, "SN1987A gamma-ray limits on the conversion of pseudoscalars," Phys. Lett. B 383 (1996) 439 [astro-ph/9605197].

[27] J. A. Grifols, E. Masso and R. Toldra, "Gamma-rays from SN1987A due to pseudoscalar conversion," Phys. Rev. Lett. 77 (1996) 2372 [astro-ph/9606028].

[28] A. Payez, C. Evoli, T. Fischer, M. Giannotti, A. Mirizzi and A. Ringwald, "Revisiting the SN1987A gamma-ray limit on ultralight axion-like particles," JCAP 1502 (2015) 006.

[29] A. De Angelis, M. Roncadelli and O. Mansutti, "Evidence for a new light spin-zero boson from cosmological gamma-ray propagation?," Phys. Rev. D 76 (2007) 121301.

[30] D. Horns and M. Meyer, "Indications for a Pair-Production Anomaly from the Propagation of VHE Gamma-Rays," JCAP 1202 (2012) 033 [arXiv:1201.4711 [astro-ph.CO]].

[31] G. I. Rubtsov and S. V. Troitsky, "Breaks in gamma-ray spectra of distant blazars and transparency of the Universe,” JETP Lett. 100 (2014) 397 [JETP Lett. 100 (2014) 355].

[32] J. Biteau and D. A. Williams, "The extragalactic background light, the Hubble constant, and anomalies: conclusions from 20 years of TeV gamma-ray observations," arXiv:1502.04166.

[33] M. Simet, D. Hooper and P. D. Serpico, “The Milky Way as a Kiloparsec-Scale Axionscope,” Phys. Rev. D 77 (2008) 063001.

[34] M. A. Sanchez-Conde et al., "Hints of the existence of Axion-Like-Particles from the gamma-ray spectra of cosmological sources," Phys. Rev. D 79 (2009) 123511.

[35] M. Meyer, D. Horns and M. Raue, "First lower limits on the photon-axion-like particle coupling from very high energy gamma-ray observation,” Phys. Rev. D 87 (2013) 035027.

[36] F. Tavecchio, M. Roncadelli and G. Galanti, "Photons to axion-like particles conversion in Active Galactic Nuclei,” Phys. Lett. B 744 (2015) 375 [arXiv:1406.2303 [astro-ph.HE]].

[37] F. Tavecchio, M. Roncadelli, G. Galanti and G. Bonnoli, "Evidence for an axion-like particle from PKS 1222+216?,” Phys. Rev. D 86 (2012) 085036 [arXiv:1202.6529 [astro-ph.HE]].

[38] J. P. Conlon and M. C. D. Marsh, "Excess Astrophysical Photons from a 0.1-1 keV Cosmic Axion Background,” Phys. Rev. Lett. 111 (2013) 151301 [arXiv:1305.3603 [astro-ph.CO]]. 
[39] S. Angus et al., "Soft X-ray Excess in the Coma Cluster from a Cosmic Axion Background," JCAP 1409 (2014) 026 [arXiv:1312.3947 [astro-ph.HE]].

[40] D. Kraljic, M. Rummel and J. P. Conlon, "ALP Conversion and the Soft X-ray Excess in the Outskirts of the Coma Cluster," JCAP 1501 (2015) 011 [arXiv:1406.5188 [hep-ph]].

[41] J. Redondo and A. Ringwald, "Light shining through walls," Contemp. Phys. 52 (2011) 211.

[42] K. Ehret et al. [ALPS Collaboration], "New ALPS Results on Hidden-Sector Lightweights," Phys. Lett. B 689 (2010) 149 [arXiv:1004.1313 [hep-ex]].

[43] R. Ballou et al. [OSQAR Collaboration], "Latest Results of the OSQAR Photon Regeneration Experiment for Axion-Like Particle Search,” arXiv:1410.2566 [hep-ex].

[44] R. Bähre et al. [ALPS Collaboration], “Any light particle search II - Technical Design Report," JINST 8 (2013) T09001 [arXiv:1302.5647 [physics.ins-det]].

[45] F. Hoogeveen and T. Ziegenhagen, "Production and detection of light bosons using optical resonators," Nucl. Phys. B 358 (1991) 3.

[46] P. Sikivie, D. B. Tanner and K. van Bibber, "Resonantly enhanced axion-photon regeneration," Phys. Rev. Lett. 98 (2007) 172002 [hep-ph/0701198 [HEP-PH]].

[47] P. Sikivie, "Experimental Tests of the Invisible Axion,” Phys. Rev. Lett. 51 (1983) 1415 [Erratum-ibid. 52 (1984) 695].

[48] S. Andriamonje et al. [CAST Collaboration], "An Improved limit on the axion-photon coupling from the CAST experiment," JCAP 0704 (2007) 010 [hep-ex/0702006].

[49] M. Arik et al. [CAST Collaboration], "CAST solar axion search with ${ }^{3} \mathrm{He}$ buffer gas: Closing the hot dark matter gap,” Phys. Rev. Lett. 112 (2014) 091302 [arXiv:1307.1985 [hep-ex]].

[50] E. Armengaud et al. [IAXO Collaboration], "Conceptual Design of the International Axion Observatory (IAXO),” JINST 9 (2014) T05002 [arXiv:1401.3233 [physics.ins-det]].

[51] S. J. Asztalos et al. [ADMX Collaboration], "A SQUID-based microwave cavity search for dark-matter axions,” Phys. Rev. Lett. 104 (2010) 041301 [arXiv:0910.5914 [astro-ph.CO]].

[52] D. Horns, A. Lindner, A. Lobanov and A. Ringwald, "WISP Dark Matter eXperiment and Prospects for Broadband Dark Matter Searches in the $1 \mu \mathrm{eV}-10 \mathrm{meV}$ Mass Range," arXiv:1410.6302 [hep-ex].

[53] G. Rybka et al., "Search for dark matter axions with the Orpheus experiment," Phys. Rev. D 91 (2015) 1, 011701 [arXiv:1403.3121 [physics.ins-det]].

[54] D. Horns, J. Jaeckel, A. Lindner, A. Lobanov, J. Redondo and A. Ringwald, "Searching for WISPy Cold Dark Matter with a Dish Antenna,” JCAP 1304 (2013) 016.

[55] D. Budker et al., "Cosmic Axion Spin Precession Experiment (CASPEr)," Phys. Rev. X 4 (2014) 021030 [arXiv:1306.6089 [hep-ph]].

[56] P. W. Graham and S. Rajendran, "New Observables for Direct Detection of Axion Dark Matter," Phys. Rev. D 88 (2013) 035023 [arXiv:1306.6088 [hep-ph]].

[57] L. Krauss et al., “Calculations for Cosmic Axion Detection,” Phys. Rev. Lett. 55 (1985) 1797.

[58] R. Barbieri et al., "Axion To Magnon Conversion: A Scheme for The Detection of Galactic Axions," Phys. Lett. B 226 (1989) 357.

[59] G. Carugno and G. Ruoso, "QUaerere AXion - A proposal for a search of galactic axions using magnetized materials," October 8, 2014. 